\title{
A novel aortic clamp distributing equal pressure along the jaws
}

\author{
Bartosz Rylski, MD, ${ }^{\mathrm{a}, \mathrm{b}}$ Claudius Schmid, MD, ${ }^{\mathrm{a}}$ Friedhelm Beyersdorf, MD, PhD, ${ }^{\mathrm{a}}$ and Matthias Siepe, $\mathrm{MD}^{\mathrm{a}}$ \\ Freiburg, Germany
}

From the ${ }^{\mathrm{a}}$ Department of Cardiovascular Surgery, Heart Center Freiburg University, ${ }^{\mathrm{b}}$ Faculty of Medicine, University Freiburg, Freiburg, Germany.

B.R. and C.S. contributed equally to this article.

Disclosures: Bartosz Rylski is a consultant to Gebrüder Martin GmbH \& Co. KG, Tuttlingen, Germany. All other authors have nothing to disclose with regard to commercial support.

Received for publication Feb 4, 2017; revisions received March 25, 2017; accepted for publication April 19, 2017; available ahead of print June 17, 2017.

Address for reprints: Bartosz Rylski, MD, Department of Cardiovascular Surgery, Heart Center Freiburg University, Hugstetter Str. 55, Freiburg 79106, Germany (E-mail: bartosz.rylski@universitaets-herzzentrum.de).

J Thorac Cardiovasc Surg 2017;154:1524-5

$0022-5223 / \$ 36.00$

Copyright (c) 2017 by The American Association for Thoracic Surgery

http://dx.doi.org/10.1016/j.jtcvs.2017.04.078

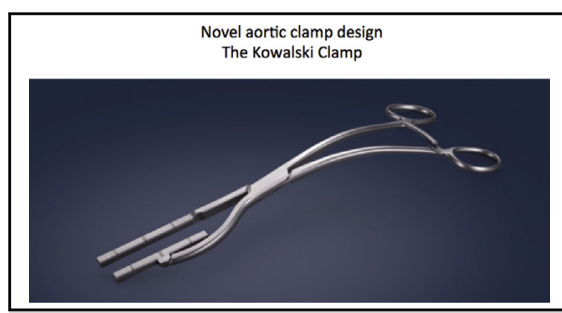

The Kowalski clamp with its additional hinge allowing the jaw to adjust its position.

\section{Central Message}

The pressure along the clamp jaws now in use is unequally distributed. We designed the novel Kowalski clamp with an additional hinge providing equal pressure distribution along the clamp jaws.

See Editorial Commentary page 1526.
We recently published evidence showing that the pressure along the clamp jaws of all the currently available vascular clamps is distributed unequally. ${ }^{1}$ This may trigger a local dissection, potentially leading to later aortic rupture. ${ }^{2-4}$ Unequal pressure distribution compels the surgeon to apply greater total clamp force to close the aorta than would be necessary were the pressure equally distributed.

To improve the clamp's construction, we designed a novel one with an additional hinge. The Kowalski clamp (named after its designer) is constructed to eliminate the unequal pressure distribution over the clamp jaws, because the additional hinge allows the upper jaw to adjust its position according to the pressure and thus provide the identical pressure in the proximal and distal quartiles (Figure 1, Video 1). The aim of this study was to measure the pressure along the jaws of the novel Kowalski clamp and compare it with the pressure measured along the currently available clamp.

\section{MATERIALS AND METHODS}

Details on an in-vitro model for pressure along the clamp jaws analysis have been described. ${ }^{1}$ To summarize, 8 descending thoracic aortas (diameter 2.0-3.0 cm) were obtained from pigs and connected to an infusion bag under pressure. Aortas were clamped with currently available vascular clamps and the novel Kowalski clamp. Each aorta was crossclamped 6 times. Aortas were filled with Sterofundin (B. Braun Melsungen AG, Melsungen, Germany), under $100 \mathrm{~mm} \mathrm{Hg}$ pressure and were clamped only as tight as necessary till no leakage at the distal aortic end was observed. The clamping pressure was measured with a pressure-detecting film (FUJI PRESCALE FILM; Fujifilm Europe GmbH, Düsseldorf, Germany). The clamped aortic segments were divided into 4 quartiles (proximal, middle proximal, middle distal, and distal to the clamp hinge). The maximum pressure required in each section was measured.

\section{RESULTS}

The pressure along the clamps jaws was distributed unequally in all the clamps except the Kowalski clamp. The

\section{Currently available clamps}

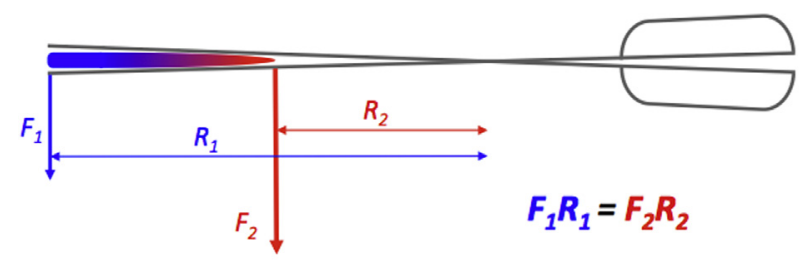

Kowalski clamp

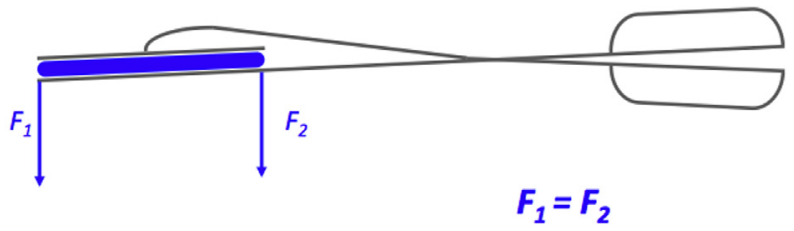

FIGURE 1. Pressure distribution in the currently available and in the Kowalski clamp. According to the law of the lever, the aorta near the hinge is under more pressure than distal to the hinge in currently available clamps. The additional hinge in the Kowalski clamp allows the upper jaw to adjust its position according to the changing pressure and thus provide the same pressure in the proximal and distal quartiles. Pictures of all tested clams are available in reference. ${ }^{1}$ 


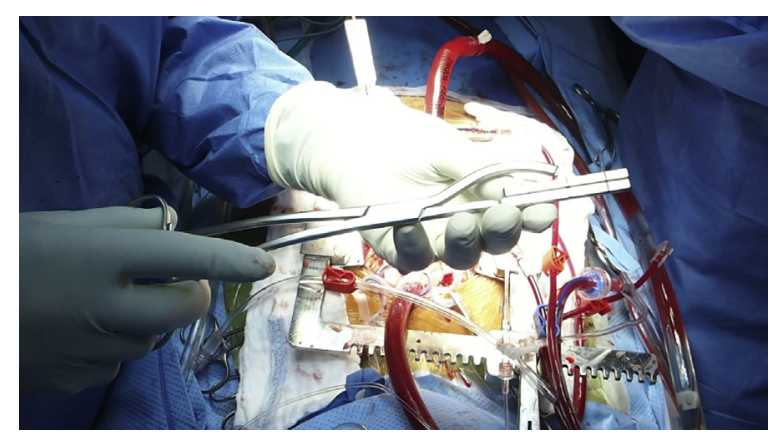

VIDEO 1. Aortic crossclamping with Kowalski clamp in a patient undergoing aortic valve replacement. Video available at: http://www.jtcvsonline. org/article/S0022-5223(17)31100-5/addons.

highest ratio between the highest and lowest pressure measured along the clamp jaws was observed in the iliac clamp (3.54). There was almost no difference (ratio 1.01) in the pressure measured along the Kowalski clamp in the proximal and distal quartiles. The Kowalski clamp demonstrated the lowest average pressure along the jaws $(0.78 \pm 0.08 \mathrm{MPa}$, Figure 2$)$.

\section{DISCUSSION}

We recently have experienced one immediate and one late contained aortic rupture 2 months after descending thoracic aortic replacement. ${ }^{2}$ Both were caused by crossclamping during the initial surgery, and the aortic wall injury in both was detected in the aortic area most proximal to the aortic clamp hinge, where according to the law of the lever the clamp force is the greatest (Figure 1). This situation motivated us to measure the pressure distribution along the jaws of the vascular clamps we routinely use. We documented unequal pressure distribution in all the vascular clamps we use in an in vitro model. ${ }^{1}$

To address the unequal pressure distribution along the clamp jaws, we designed a novel aortic clamp with an additional hinge. It is constructed to provide a homogeneous distribution of the clamping force, because the second hinge allows the upper jaw to adapt its position to the pressure. To obtain the best pressure distribution, it is essential to place the clamp so that the aorta is in the middle of the jaws (Video 1). We have added 3 short lines at both clamp jaws for better orientation in the middle of the jaws. Because aortas have different diameters, the Kowalski clamp comes in 3 sizes, each designed to accommodate the length of the jaw on the additional hinge. Size small (jaw length $5 \mathrm{~cm}$ ) is appropriate for aortas with diameter up to $3 \mathrm{~cm}$, medium $(6.5 \mathrm{~cm})$ to $4 \mathrm{~cm}$, and large $(9 \mathrm{~cm})$ to $5.5 \mathrm{~cm}$.

\section{Maximal pressure distribution generated at four different sites}

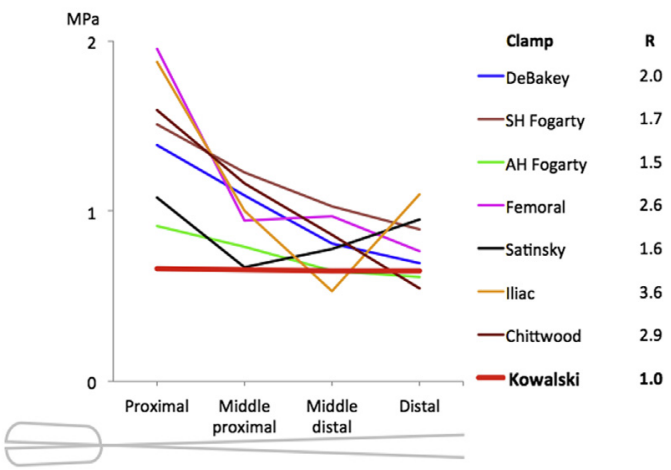

FIGURE 2. Maximum pressure along the clamp jaws after complete aortic occlusion. The proximal quartile is near, the distal quartile far from the clamp hinge. $R$, Ratio between the highest and lowest pressure measured along the clamp jaws; $S H$, straight handle; $A H$, angled handle. Data of all other clamps are obtained from Rylski and colleagues. ${ }^{1}$

Our results from this in vitro study reveal the Kowalski clamp performing superbly, offering perfectly equal distribution of the pressure along the clamp jaws. The limitation of this study is aortic model without atherosclerosis or calcification, which are sometimes observed in reality.

\section{CONCLUSIONS}

In contrast to all other available aortic clamps, the Kowalski clamp has an equal pressure distribution along the jaws. The clamp's design with an additional hinge might minimize aortic crossclamp-derived injury thereby.

We hereby acknowledge and commend Mr Leszek Kowalski for designing this novel vascular clamp with an additional hinge to ensure equal pressure distribution along the clamp jaws. We are grateful to Janet Ruprecht, Volker Scheu, Joerg Fox, and Massimo Fiamma (Gebrüder Martin GmbH \& Co.KG, Tuttlingen, Germany) for their enthusiasm in developing the Kowalski-Clamp prototype and bringing it onto the market.

\section{References}

1. Rylski B, Schmid C, Beyersdorf F, Kari FA, Kondov S, Lutz L, et al. Unequal pressure distribution along the jaws of currently available vascular clamps: do we need a new aortic clamp? Eur J Cardiothorac Surg. 2016;49:1671-5.

2. Rylski B, Beyersdorf F, Czerny M, Siepe M. Two aortic ruptures in two monthsrole of cross-clamp-associated late injury. Ann Vasc Surg. 2016;32:129.e17-19.

3. Rylski B, Hoffmann I, Beyersdorf F, Suedkamp M, Siepe M, Nitsch B, et al. Iatrogenic type A aortic dissection: insight from the German Registry for Acute Aortic Dissection Type A (GERAADA). Eur J Cardiothorac Surg. 2013;44:353-9. 4. Babin-Ebell J, Gimpel-Henning K, Sievers HH, Scharfschwerdt M. Influence of clamp duration and pressure on endothelial damage in aortic cross-clamping. Interact CardioVasc Thorac Surg. 2010;10:168-71. 